\title{
Acoustic Intensity Fluctuations Induced by South China Sea Internal Tides and Solitons
}

\author{
Ching-Sang Chiu, Steven R. Ramp, Christopher W. Miller, Member, IEEE, James F. Lynch, Senior Member, IEEE, \\ Timothy F. Duda, and Tswen Yung Tang
}

\begin{abstract}
Between late April and May 23, 2001, a suite of acoustic and oceanographic sensors was deployed by a team of U.S., Taiwan, and Singapore scientists in the northeastern South China Sea to study the effects of ocean variability on low-frequency sound propagation in a shelfbreak environment. The primary acoustic receiver was an $L$-shaped hydrophone array moored on the continental shelf that monitored a variety of signals transmitted along and across the shelfbreak by moored sources. This paper discusses and contrasts the fluctuations in the $400-\mathrm{Hz}$ signals transmitted across the shelfbreak and measured by the vertical segment of the listening array on two different days, one with the passage of several huge solitons that depressed the shallow isotherms to near the sea bottom and one with a much less energetic internal wavefield. In addition to exhibiting large and rapid temporal changes, the acoustic data show a much more vertically diffused sound intensity field as the huge solitons occupied and passed through the transmission path. Using a space-time continuous empirical sound-speed model based on the moored temperature records, the observed acoustic intensity fluctuations are explained using coupled-mode physics.
\end{abstract}

Index Terms-Intensity fluctuations, nonlinear internal waves, shallow-water acoustics, South China Sea (SCS).

\section{INTRODUCTION}

I N LATE April and May 2001, as a main component of the Asian Sea International Acoustics Experiment (ASIAEX), a suite of environmental and acoustic moorings and towed instruments were deployed by a team of U.S., Taiwan, and Singapore scientists on a shelf-edge site in the northeastern (NE) South China Sea (SCS) to simultaneously measure the ocean variability and the associated fluctuations in acoustic propagation at low frequencies [1]. Distributed primarily along two orthogonal acoustic transmission paths, the environmental moorings were designed to observe the physical oceanographic variability on time scales from internal-wave (minutes) to subtidal (days) periods. Acoustic data were primarily taken by an L-shaped hydrophone array moored on the shelf. This listening array consisted of 16 hydrophones moored vertically in the water column and 32 hydrophones spanning approximately half a kilometer horizontally along the sea floor. Sampling continuously at a rate

Manuscript received May 23, 2003; revised November 19, 2004. This work was supported by the U.S. Office of Naval Research.

C.-S. Chiu, S. R. Ramp, and C. W. Miller are with the Oceanography Department, Naval Postgraduate School, Monterey, CA 93943 USA (e-mail: chiu@nps.navy.mil).

J. F. Lynch and T. F. Duda are with the Woods Hole Oceanographic Institution, Woods Hole, MA 02543 USA.

T. Y. Tang is with the National Taiwan University, Taipei, 106 Taiwan.

Digital Object Identifier 10.1109/JOE.2004.834173 of $3.2 \mathrm{kHz}$ over the first $3 \mathrm{wk}$ in May, these hydrophones monitored repetitive phase and frequency-modulated signals transmitted from both fixed and towed sources. The fixed sources have transmission frequencies ranging from 224 to $500 \mathrm{~Hz}$ and were moored on a slope and a shelf location to define the crossand along-shelf transmission path, respectively.

The ASIAEX SCS was a progression from previous similar coupled physical oceanography and acoustics experiments, namely Shallow-Water Acoustic Random Media (SWARM) [2] and Shelfbreak PRIMER [3], with the objective to expand and advance the understanding of the physics, variability, and predictability of low-frequency sound propagation in a shelf-slope environment. With the addition of an L-shaped array, an increased number of sources covering diverse frequencies and the usage of multiple Taiwanese research vessels, the measurement advancements in ASIAEX SCS have included the horizontal properties of the sound field in addition to the vertical properties, signal transmissions covering the entire low frequency band of up to $600 \mathrm{~Hz}$, and simultaneous acoustic and oceanographic time series spanning a full spring-neap tidal cycle. The latter is necessary to support a full characterization of the temporal ocean and acoustic variability induced by the internal tides and nonlinear internal waves. Furthermore, the addition of a new transmission orientation, i.e., the along-shelf path, has provided new type of data containing information on the anisotropy of the shelf-edge acoustic wavefield.

Density and sound-speed fluctuations of the SCS shelf-slope region in May 2001 were observed to be dominated by "transbasin" and local internal tides and by "transbasin" nonlinear internal waves generated remotely in Luzon Strait via shallow ridges-tidal current interactions during spring and neap tides. The shoaling water depth amplified the disturbances of these nonlinear internal waves as they evolved shoreward onto the shelf. The scattering of these transbasin waves by the nearby Dongsha Island also contributed local waves to the composition of the nonlinear internal wavefield in the experimental site. A detailed discussion of these oceanographic conditions can be found in Ramp et al. [4]. The impacts of these nonlinear internal waves on various aspects of the acoustic propagation are the subject of discussion in several papers of this volume: Orr et al. [5] investigate the impact on horizontal coherence and horizontal beamforming performance and Duda et al. [6] examine the resultant characteristic features in the intensity-fluctuation time series and compare the signal statistics between the along- and cross-shelf paths. In this paper, the focus is on explaining and contrasting the observed intensity fluctuations of the $400-\mathrm{Hz}$ cross-shelf transmissions in two separate days having extreme 


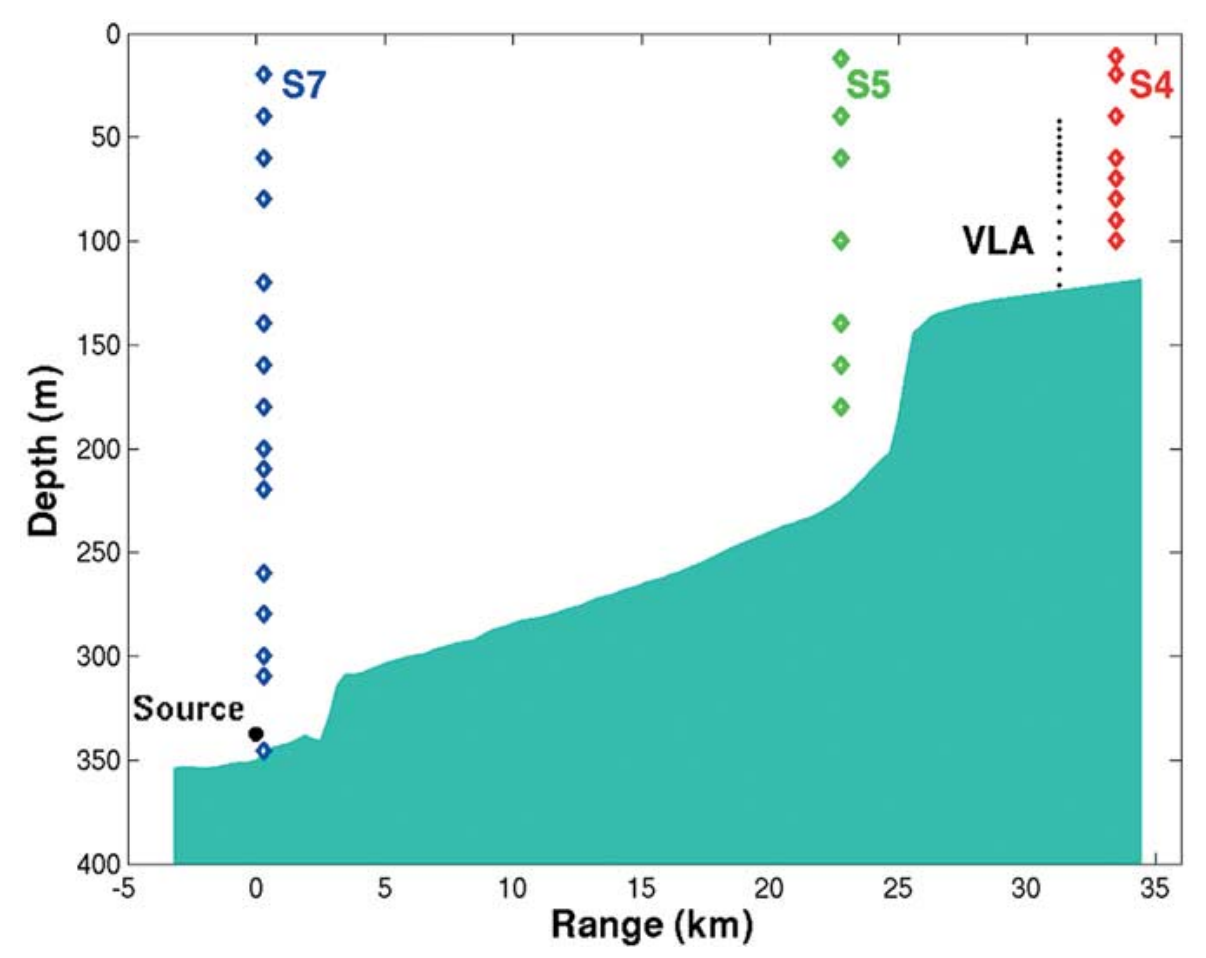

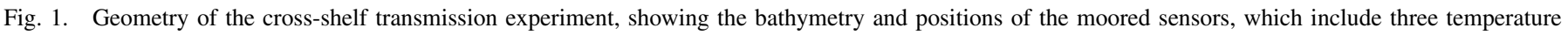

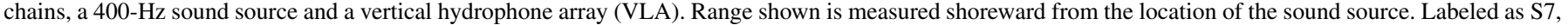
$\mathrm{S} 5$, and S4, the temperature moorings are located at the 346-, 226-, and 120-m isobaths, respectively.

environmental differences: one (May 8) with the passage of several huge solitons that depressed the shallow isotherms to the sea bottom and the other (May 4) with a much less energetic internal wavefield. The internal tides, however, dominated the sound-speed variability on May 4. Specifically, the interpretation of the observed changes in the vertical distribution of sound intensity is aided with coupled-mode propagation modeling facilitated with a space-time continuous empirical representation of the sound-speed field.

The outline of this paper is as follows. We begin in Section II with an examination of the space-time structure of the soundspeed variability along the cross-shelf transmission path. The temporal and spatial structures of the sound-speed field were analyzed from the moored temperature data using temporal filtering to first delineate the perturbations in the subtidal, tidal, and supertidal bands, followed by principle-component decompositions to capture dominant vertical structures. The aims here are to expose the differences between May 4 and 8, and to arrive at an efficient mathematical representation of the sound-speed changes. In Section III, the observed sound intensities across the vertical aperture of the array for May 4 and 8 are presented and discussed. In Section IV, the intensity changes and differences are further explored by comparison with propagation modeling results. The empirical model that we used to approximate the evolution of sound speed in finite time windows for the acoustic modeling is also presented in this section. The conclusion of this paper is offered in Section V.

\section{SOUND-SPEED STRUCTURES}

The cross-shelf transmission path was occupied with three environmental moorings containing vertically distributed ther- mometers (some of which also sampled salinity). Called S7, S5, and S4, they were moored at the 348-, 226-, and 120-m isobaths, respectively. The locations of these environmental moorings relative to the positions of the $400-\mathrm{Hz}$ sound source and listing array and to the local bathymetry are depicted in Fig. 1. The bathymetry was first mapped by a "pilot" site survey carried out in June 2000 [7] and then improved with additional bathymetric (i.e., echo sounder) data collected along the ASIAEX SCS shiptracks. The depths of each of the thermometers, the source and each of the hydrophones that formed the vertical line array (VLA), i.e., the vertical segment of the L-shaped array, are also depicted in Fig. 1. Sampling every minute from April 23 to May 18, the temperature records were converted into soundspeed time series using an empirical formula [8] with a nominal salinity value.

Our procedure to characterize the multiscale sound-speed variability began with filtering the time series from each mooring into a mean profile $\bar{c}$ plus three perturbation components, i.e.,

$$
\begin{aligned}
c(r, z, t)=\bar{c}(r, z)+\delta c_{M}( & r, z, t) \\
& +\delta c_{T}(r, z, t)+\delta c_{\mathrm{IW}}(r, z, t)
\end{aligned}
$$

where $\delta c_{M}, \delta c_{T}$, and $\delta c_{\mathrm{IW}}$ are the variations caused by mesoscale ocean processes, internal tides, and internal waves in nonoverlapping frequency bands, dubbed in this paper as subtidal, tidal, and supertidal, respectively. In (1), $r$ is the mooring's horizontal distance (range) from the sound source, $z$ is depth, and $t$ is time. For each frequency band $i$ and for each mooring location $r$, the procedure then decomposed the sound-speed perturbation time series into a linear combination 


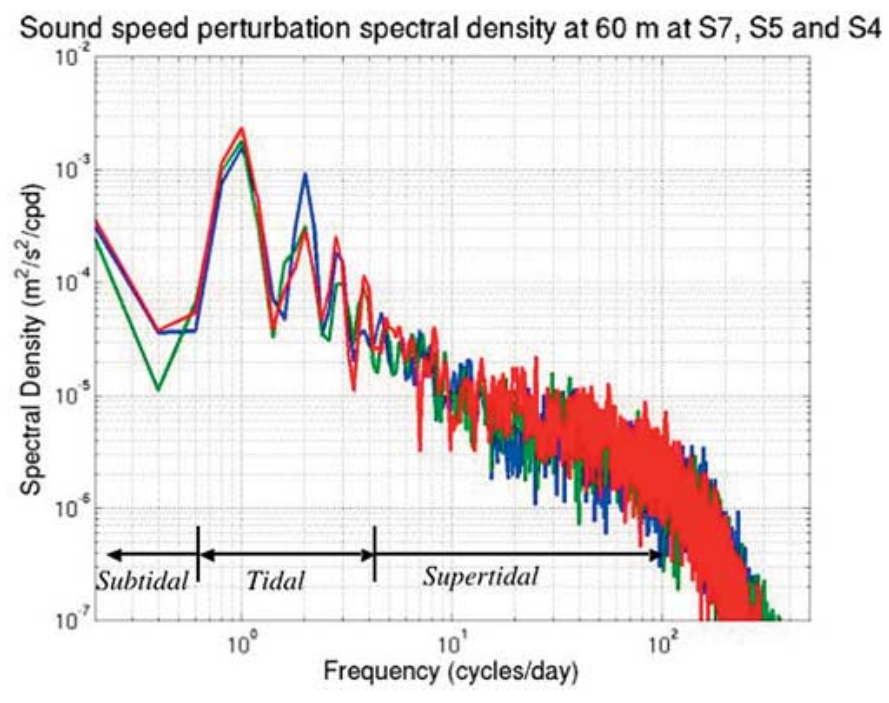

Fig. 2. Spectral densities of sound-speed perturbations measured at a depth of $60 \mathrm{~m}$ at the 346-m (blue), 226-m (green), and 120-m (red) isobaths, respectively.

of vertical, empirical orthogonal functions (EOFs) or modes $f_{n}^{(i)}$, weighted by the corresponding amplitude time series $a_{n}^{(i)}$

$$
\begin{aligned}
\delta c_{i}(r, z, t) & =\sum_{n=1}^{N} a_{n}^{(i)}(r, t) f_{n}^{(i)}(z ; r) \\
i & =M, T, \mathrm{IW} .
\end{aligned}
$$

While the temporal decomposition (1) was accomplished with Butterworth filters, the EOFs and their amplitudes were obtained using principal-component analysis followed by projecting the data onto the EOFs.

The selection of the proper frequency bands to delineate the different physical scales was guided with observed spectral characteristics in the sound-speed time series. Fig. 2 shows the spectral densities of the time series at a depth of $60 \mathrm{~m}$ for each of the three moorings. (Note that a color scheme of blue, green, and red is used consistently in all the figures of this section to denote analyzed data from the S7, S5, and S4 moorings, respectively.) Next to a local minimum at about $0.5 \mathrm{cpd}$, prominent spectral peaks are clearly shown at approximately 1, 2, 3 , and $4 \mathrm{cpd}$, which are then followed by a diffused spectrum. The spectral peaks are associated with diurnal and semidiurnal internal tides and the higher order tidal harmonics, whereas the diffused spectrum is associated with a continuum of higher frequency internal waves. Accordingly, the subtidal, tidal, and supertidal bands were chosen to be periods of longer than 2 $\mathrm{d}$, between $5 \mathrm{~h}$ and $2 \mathrm{~d}$ and shorter than $5 \mathrm{~h}$, respectively. The analyzed structures of the mean and perturbation fields are discussed next.

Fig. 3 shows the mean sound-speed profiles at each of the three mooring locations, S7, S5, and S4. The three profiles are seen to lie on top of each other. This indicates that the mean sound-speed field was range independent and that a shelfbreak front did not exist during this April-May time frame in 2001. A clear shelfbreak front, however, was observed by an oceanographic survey conducted during the same months in the previous year [9]. This front is nominally created during wintertime by the intrusion of warmer Kuroshio water in the western North

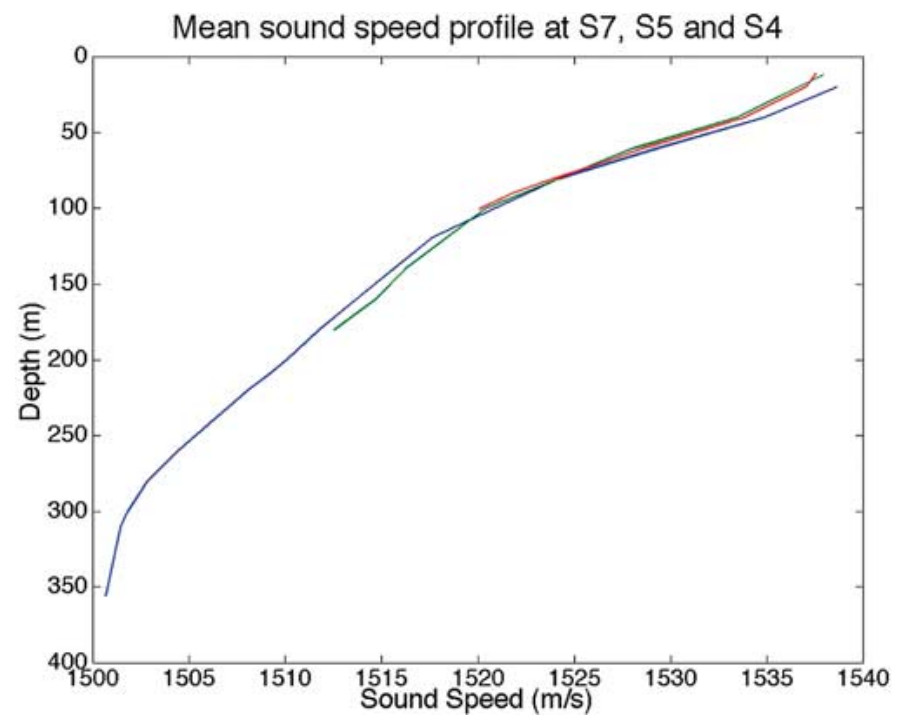

Fig. 3. Time-averaged sound-speed profiles measured at the 346-m (blue), 226-m (green), and 120-m (red) isobaths.

Pacific into the NE SCS as a result of the prevailing winter monsoon wind. April-May is nominally the break period between the winter and summer monsoons. Gawarkiewicz et al. [9] explains that the timing and duration of the shelfbreak front depends on the onset of the winter monsoon. The winter monsoon arrived earlier in 2001 and the intrusion water retreated before April-May.

Results from the vertical decompositions (2) show that empirical orthogonal modes are an efficient representation of the cross-shelf sound-speed perturbations. In each of the three frequency bands, only two modes are necessary to capture $77 \%-95 \%$ of the variances. Moreover, the first mode alone consistently accounts for about $70 \%$ of the variance. Fig. 4 shows the first two empirical vertical modes of sound-speed perturbation as a function of frequency band and mooring location, with the corresponding mode-amplitude time series displayed in Fig. 5. The modes were normalized to have unit energy. In each frequency band, the vertical structures at S7, $\mathrm{S} 5$, and $\mathrm{S} 4$ are alike, with modal maxima shoaling relative to water depth. An exception is the first internal-tide mode at S5 (i.e., near the shelfbreak) with its maximum shifted to a lower depth. Correlation estimates between the amplitude time series show that for periods $\geq 2$ days (subtidal band), the sound-speed variations at S7, S5, and S4 are highly correlated with variance slightly decreasing shoreward. This implies that, in the absence of the shelfbreak front, mesoscale processes affecting the experimental region have spatial scales much longer than the transmission path, producing approximately range-independent sound-speed changes. Good correlations are also found between the mode-amplitude time series at S7, S5, and S4 in the tidal band, but with variance increasing shoreward. This variance increase can be attributed to the shoaling bathymetry that forces the internal tide to grow in amplitude as it propagates from deep to shallow water.

Unlike its low-frequency counterparts, the supertidal soundspeed fluctuations at S7, S5, and S4 have very little correlation. Shown in the lower left panel of Fig. 5, the generally positive 

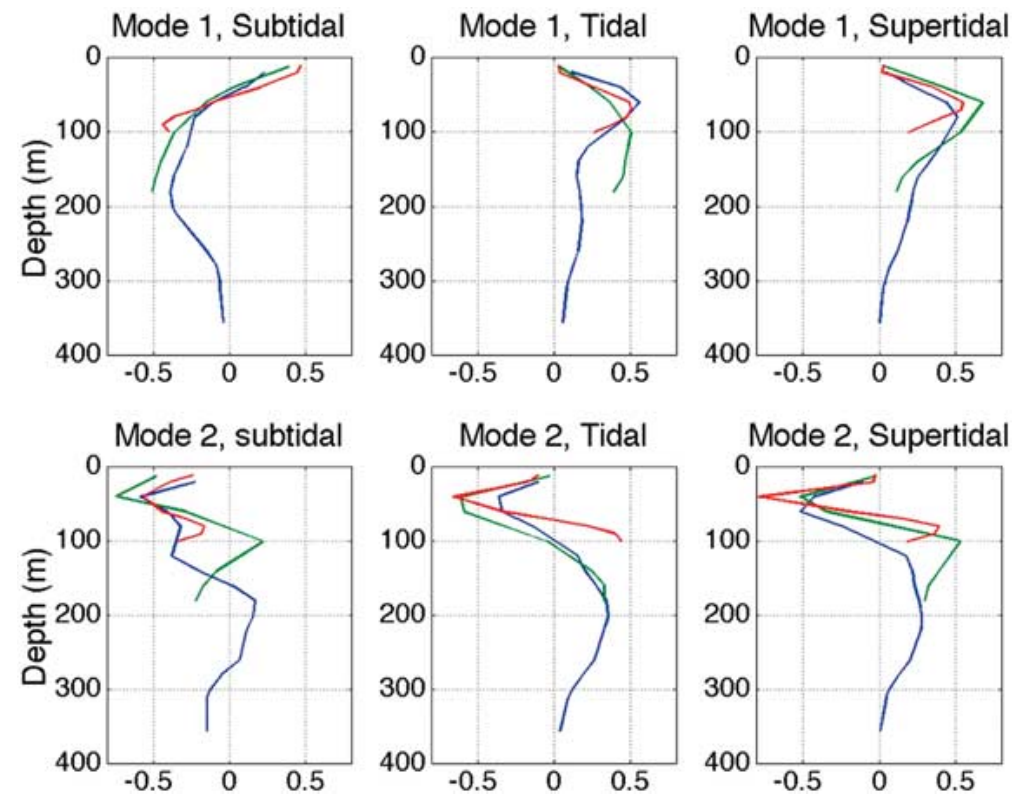

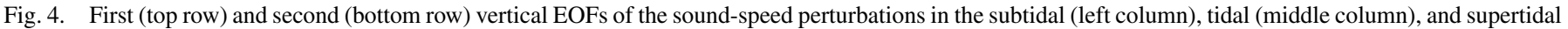
(right column) frequency bands, measured at the 346-m (blue), 226-m (green), and 120-m (red) isobaths, respectively.
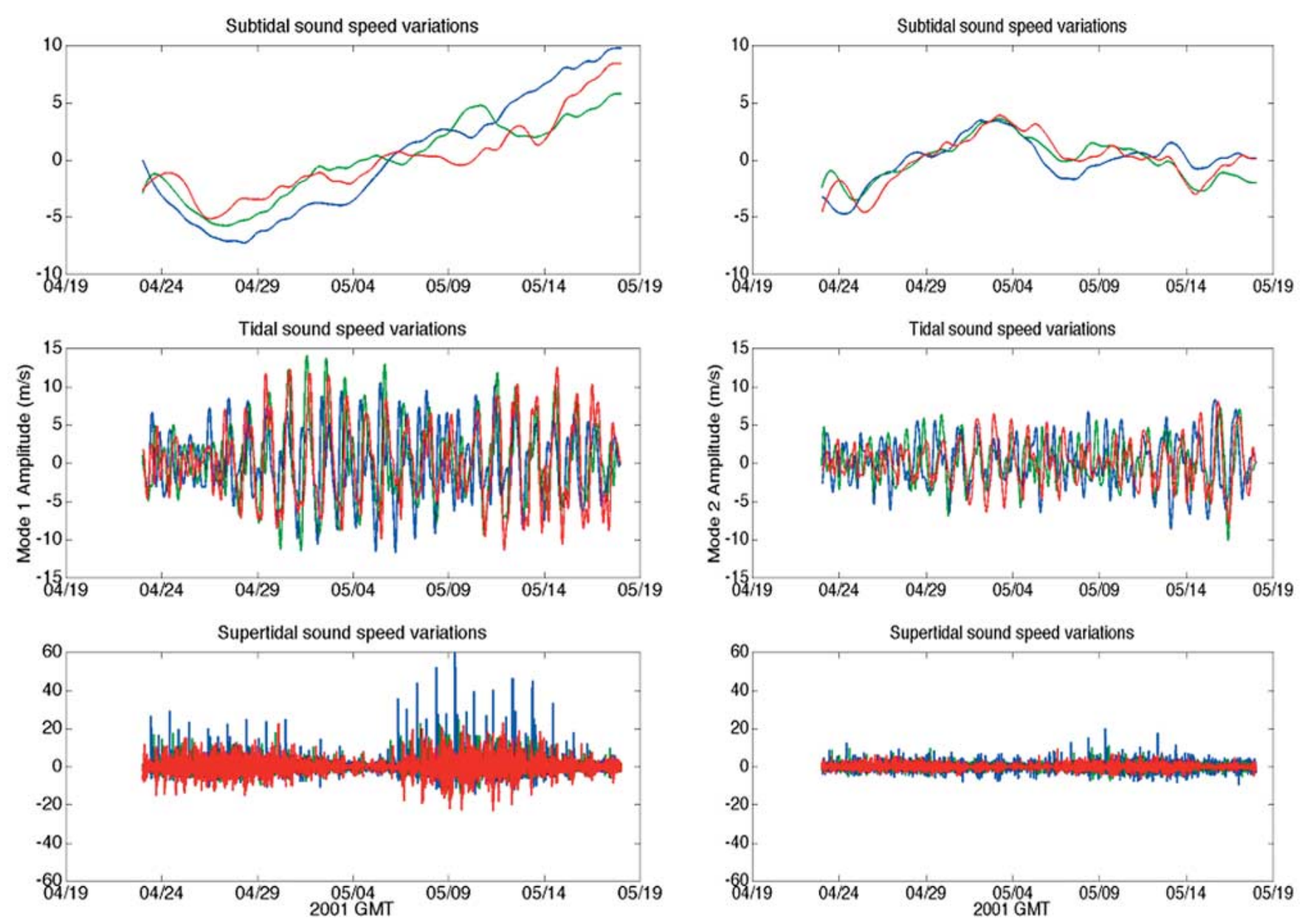

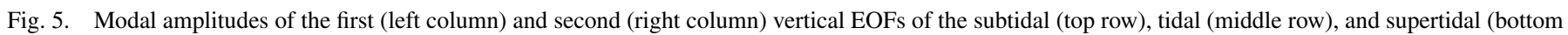
row) sound- speed perturbations, measured at the 346-m (blue), 226-m (green), and 120-m (red) isobaths, respectively.

perturbations in this band were produced by large-amplitude, nonlinear, depression internal waves, loosely referred to here as internal solitons. The lack of direct correlation can be attributed to the dispersive nature of the transbasin solitons as they 


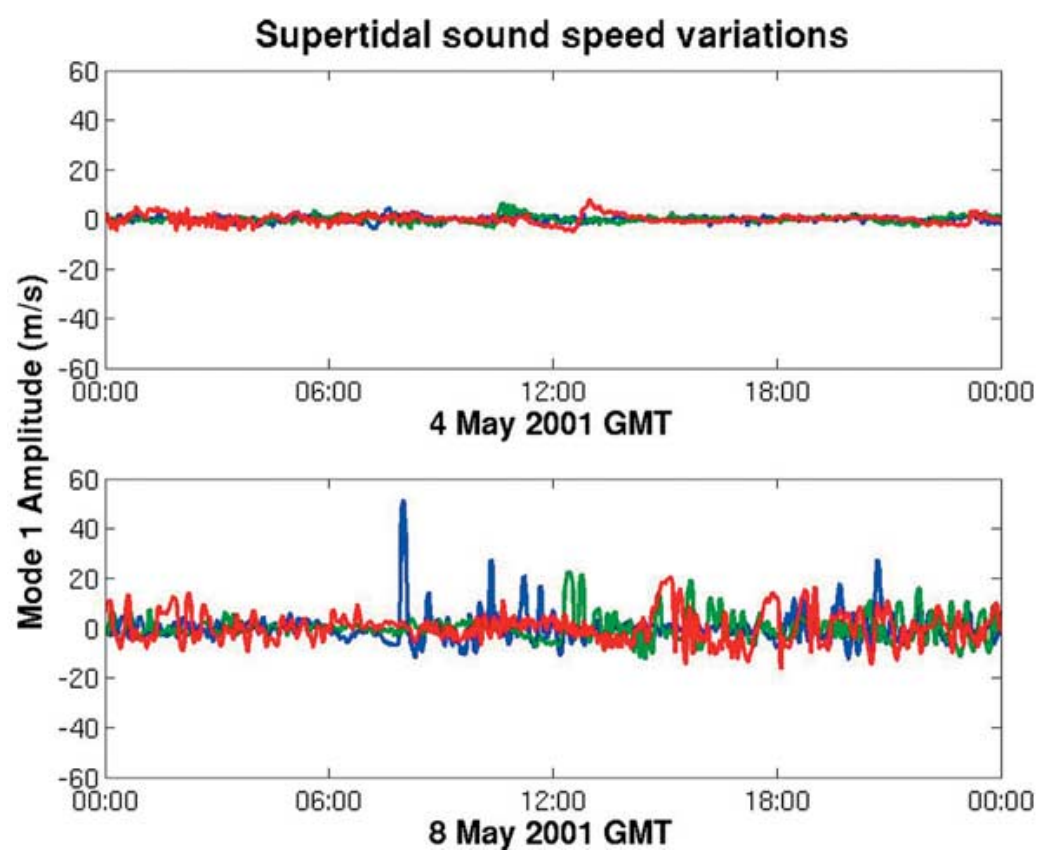

Fig. 6. Amplitudes of the first vertical EOF of the supertidal sound-speed perturbations, measured at the 346-m (blue), 226-m (green), and 120-m (red) isobaths on May 4 (top) and May 8 (bottom), respectively.

evolve shoreward and the addition of new solitons at the shelfbreak. A possible generation mechanism of the new solitons is the scattering of the transbasin waves by the nearby Dongsha Island [4]. Aside from these more subtle characteristics, two important features can be easily discerned from the time series. First, the soliton activities were time varying. There were two periods of strong activity separated by a period of no activity. The increase and decrease of the fluctuations were controlled by the spring-neap (barotropic) tidal cycle at the generation site of these transbasin waves off the Batan Islands at the entrance of the SCS [4]. In particular, the supertidal sound-speed fluctuations reached a minimum between May 4 and 5 and a maximum between May 8 and 9. Second, the internal tide-induced fluctuations tended to reach a local minimum as the soliton-induced fluctuations reached a maximum. This makes sense in an energy-conservation concept that the internal tides are generated initially and, as they propagate over the basin, part of their energy is transferred to the supertidal band through nonlinear mechanisms. Thus, reduced internal-tide amplitude is associated with increased soliton amplitude. Note that both transbasin and locally generated internal tides were observed during the experiment, but the latter did not appear until May 10 [4].

In summary, May 4 and 8 represent two very different days having radically dissimilar environmental conditions. To accentuate the difference, Fig. 6 zooms in the May 4 and 8 segments of the amplitude time series of the mode- 1 sound-speed perturbations in the supertidal band. While there were no solitons on May 4, there were huge solitons on May 8. The sound-speed variability on May 4 was dominated the internal tides. During May 8, two packets of internal solitons, separated by the semidiurnal period, are seen at S7 from Fig. 6, with the first packet led by one of the largest solitons found in the entire experiment. This leading, huge soliton introduced a maximum sound-speed change of approximately $25 \mathrm{~m} / \mathrm{s}$ (mode function times ampli- tude) at a depth of $80 \mathrm{~m}$ on the continental slope. As time of the day progresses, the packet is seen to evolve from S7 to S5 and then to $\mathrm{S} 4$ with changes in the waveform, i.e., dispersion. The most obvious change is that the widths of the solitons tend to grow as their amplitudes reduce shoreward. One can imagine that these depressions are so large that they are mostly limited by the water depths. Thus, as water depth decreases, the soliton height must reduce and a widening must then compensate this height reduction. As mentioned before, the internal waveform is further complicated by additional new solitons formed locally. Note that the actual direction of propagation of the solitons did not coincide with the orientation of the cross-shelf moorings [4]. Thus, the disturbances observed were not colocated on the crests of the waves.

In relation to the cross-shelf transmission path, the timing of the first soliton packet on May 8 is as such: the leading soliton entered the transmission path at 08:00, traveled to the shelfbreak at around 13:00, and then reached the VLA at about 15:00. Time is reported in this paper in Greenwich Mean Time (GMT). Because of the residue from the second soliton packet of the previous day, appreciable fluctuations are found along the path throughout May 8, except for a 1-h period right before the entrance of the huge soliton (Fig. 6). We label this short moment of minimal fluctuations as "the calm hour."

\section{AcOUStic InTENSITY DATA}

Moored at $13 \mathrm{~m}$ above the $350-\mathrm{m}$ isobath on the slope (Fig. 1), the sound source transmitted phase-modulated signals at a carrier frequency of $400 \mathrm{~Hz}$ with a bandwidth of $100 \mathrm{~Hz}$ and a source level SL of $180 \mathrm{~dB}$ re $1 \mu \mathrm{Pa}$. The phase modulation employed was a 5.11-s-long 511-digit pseudorandom sequence resulting in a compressed pulse of 10-ms resolution after matchedfiltering. During both May 4 and 8, a transmission of 88 consec- 


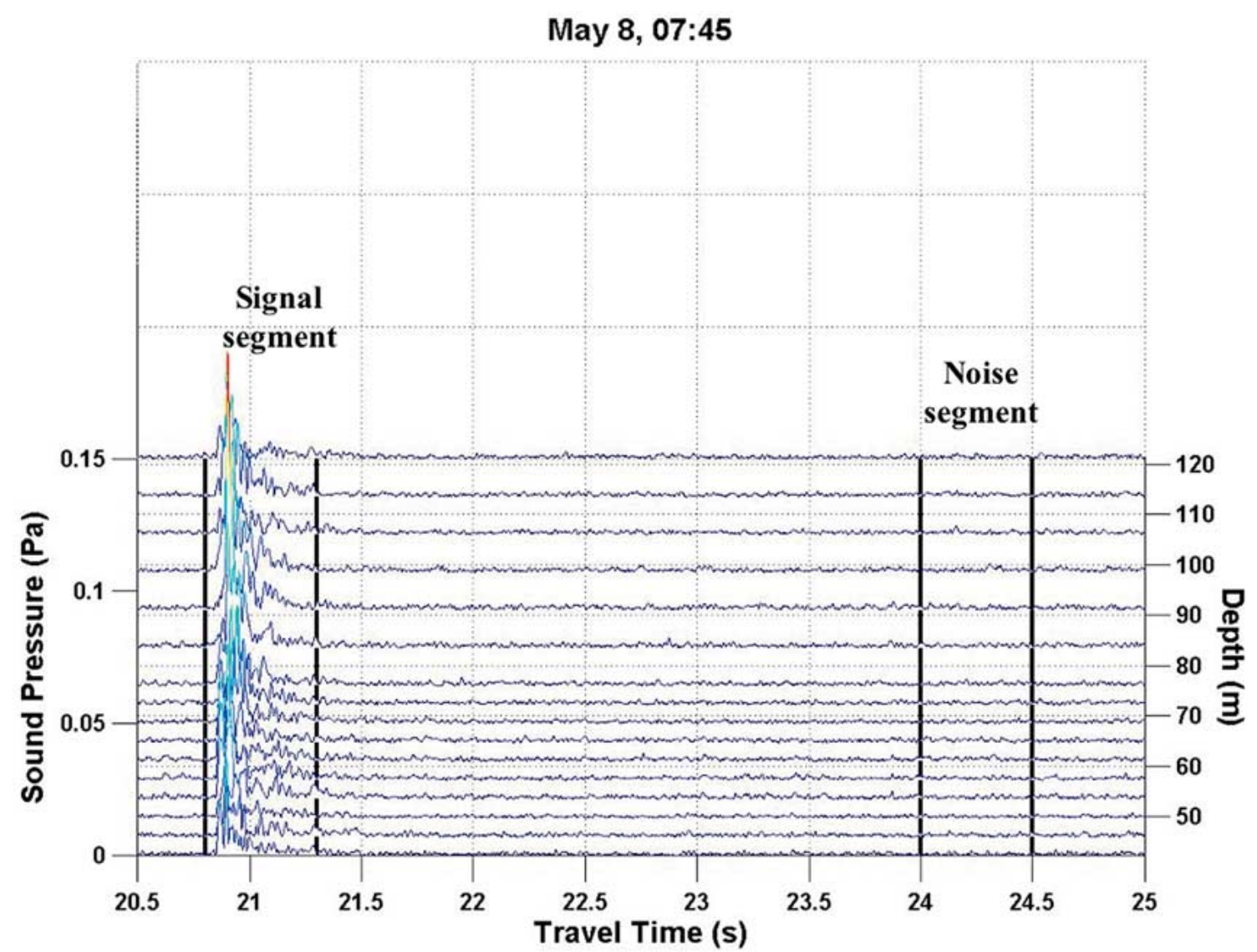

Fig. 7. Multipath arrival structure of a pulse versus depth, measured by the VLA at 07:45 on May 8. The arrival structure displayed is the magnitude the complex envelope of the matched-filter output, showing a multipath spread of approximately $0.5 \mathrm{~s}$. The transmitted signal has a bandwidth of $100-\mathrm{Hz}$, centered at $400 \mathrm{~Hz}$.

utive sequences of the signal was performed every half hour. Each transmission thus lasted about 7.5 min. These bihourly transmissions were sampled at a rate of $3.2 \mathrm{kHz}$ by the VLA moored at the 125-m isobath on the shelf. The VLA has an aperture of $79 \mathrm{~m}$ spanning the depths from 42 to $121 \mathrm{~m}$. The received signals, after matched-filter processing, correspond to the multipath arrival structure of the compressed pulse, i.e., pulse response, measured as a function of depth and time. The pulse response measured at 07:45 across the VLA aperture on May 8 , just before the huge soliton entered the transmission path, is displayed in Fig. 7. It shows a multipath spread of about $0.5 \mathrm{~s}$, which is consistent throughout the data set.

The pulse-compression scheme improved the signal-to-noise ratio (SNR) significantly (by more than $20 \mathrm{~dB}$ ) and, hence, provided high-quality estimates of the sound intensity level. In our estimate, sound intensity level is defined as

$$
\mathrm{SIL}=10 \log \left(\frac{\frac{1}{T} \int_{\text {over } T} p^{2}(t) d t}{1 \mu \mathrm{Pa}^{2}}\right)
$$

where $T$ is the multipath spread, taken to be $0.5 \mathrm{~s}$, and $p$ the signal segment in the matched-filter output. There are different ways to normalize the matched filter. We have chosen to set the division of the energy of compressed source pulse by the multipath spread $T$ equal to the power of the carrier source signal. Using this normalization, transmission loss (TL) at the
VLA location is simply the difference between SL and SIL, i.e., $\mathrm{TL}=$ SL-SIL.

Contaminated by matched-filtered noise $n$, the SIL estimate contains error $\varepsilon$

$$
\operatorname{SIL}+\varepsilon=10 \log \left(\frac{\frac{1}{T} \int_{\text {over } T}[p(t)+n(t)]^{2} d t}{1 \mu \mathrm{Pa}^{2}}\right) .
$$

An accuracy measure would be useful for assuring the quality of the SIL estimates. Through a truncated Taylor series expansion of (4) to provide a linearized relation between $\varepsilon$ and $n$, followed by squaring and then forming ensemble averages, a linearized expression for the standard deviation $\sigma_{\varepsilon}$ of $\varepsilon$ as a function of the standard deviation $\sigma_{n}$ of $n$ can be obtained. Assuming uncorrelated noise, the expression is simply

$$
\sigma_{\varepsilon}=\left(\frac{20 \log e}{\sqrt{f_{s}}}\right) \frac{\sigma_{n}}{\sqrt{\int_{\text {over } T} p^{2}(t) d t}}
$$

where $f_{s}$ is the sampling frequency of the data. Using (5) with $\sigma_{n}$ values calculated from noise segments (see Fig. 7) extracted from the matched-filter outputs, the standard deviations of the errors in the SIL estimates are found to be quite negligible, with values consistently lower than $0.1 \mathrm{~dB}$ throughout.

To make a direct visual connection to the supertidal soundspeed fluctuations displayed in Fig. 6, we first show in Fig. 8 the variances of the sound intensity levels measured over each of the 7.5-min-long transmissions across the VLA on May 4 and 8, 


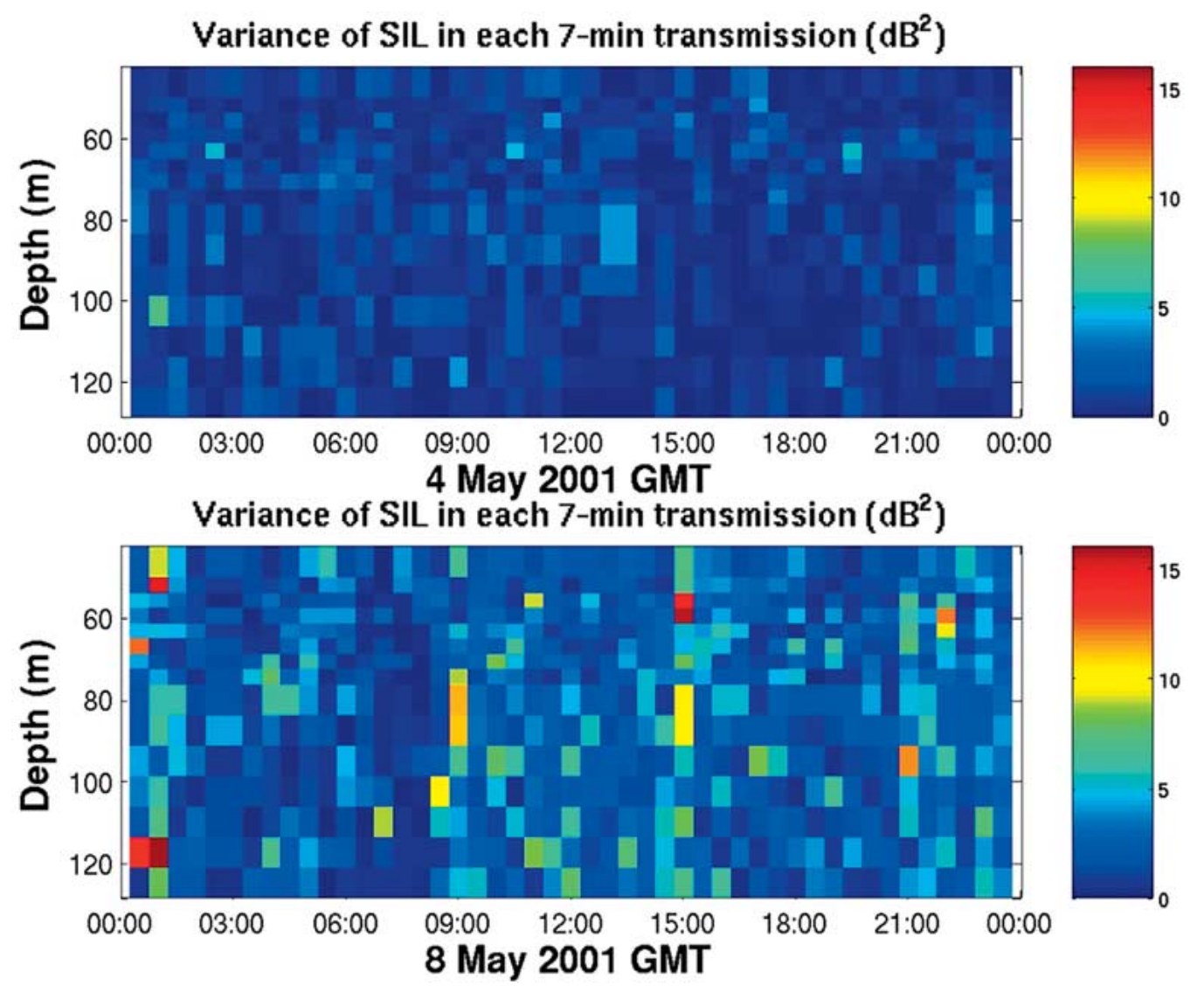

Fig. 8. Variances of the sound intensity levels measured over each of the 7.5-min-long transmissions across the VLA on May 4 (top) and 8 (bottom), respectively.

respectively. Since these are SIL variances observed over short time intervals, they are associated with internal waves only. With no soliton activity, the variances on May 4 are small, as expected. In contrast, the variances on May 8 are consistently four times higher that those of May 4, resulting from the passage of the large-amplitude solitons. It is interesting to note that the calm hour (from 07:00 to 08:00) immediately preceding the entrance of the huge soliton on May 8 stands out in the figure as a period of much reduced intensity variances.

The varying sound intensity levels themselves measured within each of the transmission periods on May 4 and 8 are color-contoured in Figs. 9 and 10, respectively, as a function of time and depth. We will call these the intensity patterns and note that the width of each vertical color strip in the figure represents $7.5 \mathrm{~min}$ in an expanded scale. The intensity pattern on May 4 is relatively smooth with the lower layer (below $70 \mathrm{~m}$ ) generally being more intense due to the downward-refracting nature of the sound channel. The pattern shows two high intensity zones that slowly fade in and out in the lower layer and one in the intermediate layer (between 42 and $70 \mathrm{~m}$ ) that cycles up and down. These fading and cycling behaviors are in strong accord to the internal tides observed near the source at S7, which alternately stretched and compressed the sound channel there. The May 4 intensity pattern also shows an anomalously silent period between 18:00 and 22:00. This, by the way, was the only period of the day in which the internal tides were observed to compress the sound-speed profiles at S4, S5, and S7 simultaneously, i.e., through out the entire path. These increased vertical gradients, especially near the VLA on the shallow shelf, might have driven most of the acoustic energy to the sediment.

In contrast, except for prior to the entrance of the huge soliton, the May- 8 intensity pattern shown in Fig. 10 has the appearance of being madly shaken, vertically. The smoothness of the pattern prior to 08:00 is, in appearance, similar to that of May 4. One can attribute this similarity to the smallness of the soliton disturbances during those early hours. Directly preceding the shaken period starting at 08:00 was the calm hour, during which the acoustic energy was mostly confined in the lower layer (below $70 \mathrm{~m}$ ). However, as the packet of huge solitons entered and moved along the acoustic path, the SIL generally decreased in the lower layer but increased in the intermediate layer until the huge solitons passed the shelfbreak at approximately 13:00. Note that the leading soliton ultimately passed the VLA at about 15:00 and that the next and less energetic soliton packet in the same day entered the acoustic path at about 20:00. Since the degree of intensity fluctuation depends inversely on bandwidth, narrowing the bandwidth can provide a more dramatic look at the impact of a huge soliton. For this, in Fig. 11 we display a "before" and "after" glance at the measured SIL in a 1-Hz bin across the VLA. Consistently, these narrow-band results show that, right before the entrance of the huge soliton, the high-intensity zone was confined below $70 \mathrm{~m}$ with a quasi-acoustic null just below $100 \mathrm{~m}$. After the entrance, the acoustic intensity was diffused/scattered into the shallower depths. The changes in these narrowband intensities, however, are seen to be much more appreciable, $10 \mathrm{~dB}$ and more. 


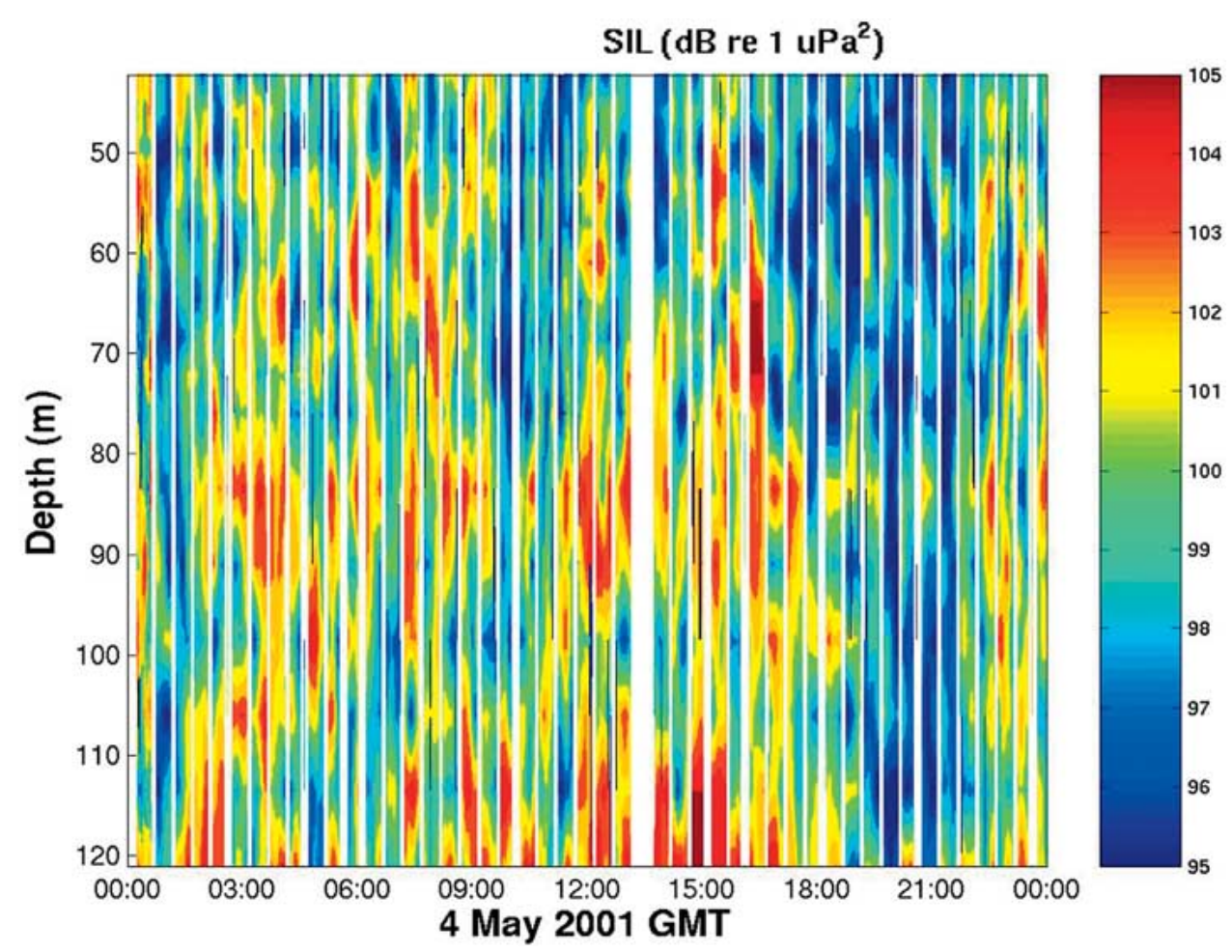

Fig. 9. Sound-intensity pattern measured by the VLA as a function of time and depth on May 4.

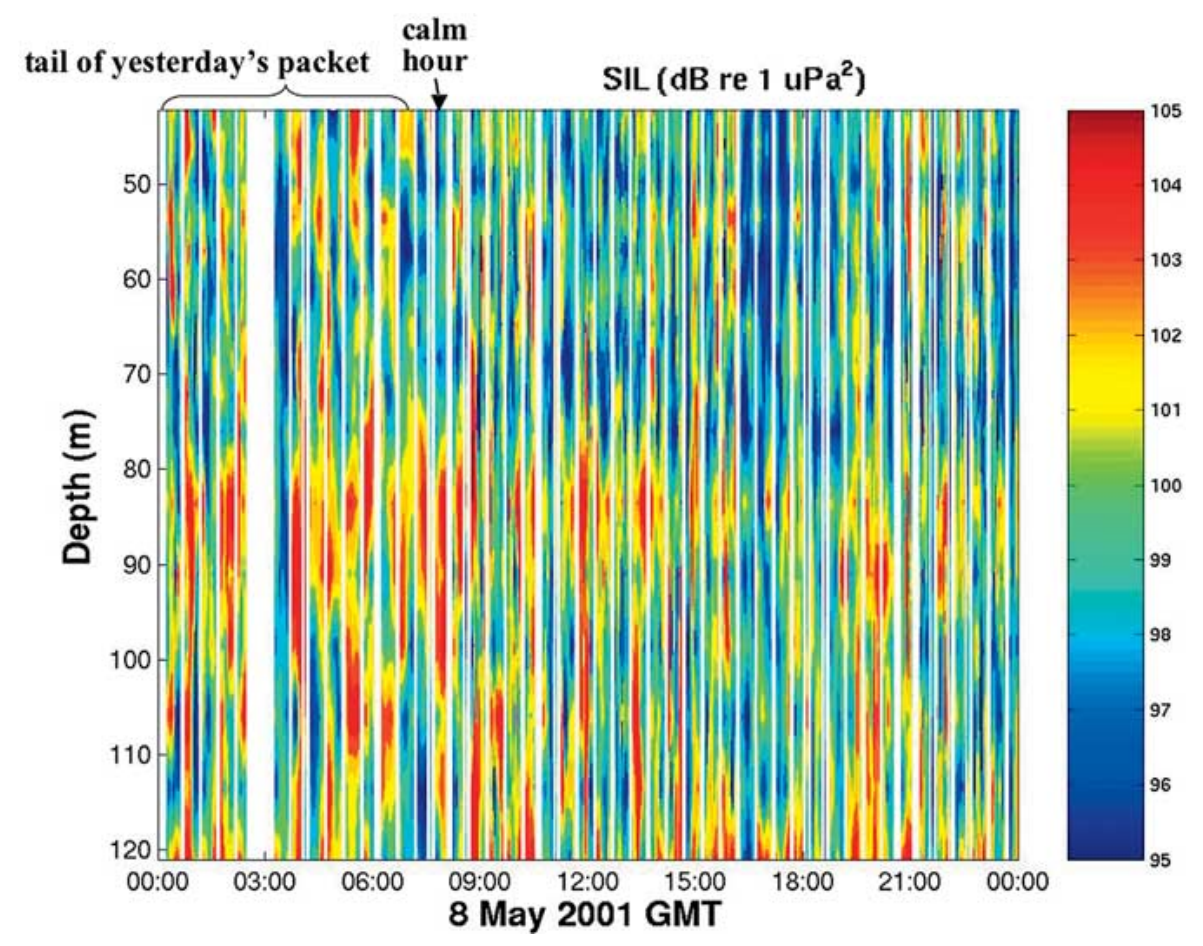

Fig. 10. Sound-intensity pattern measured by the VLA as a function of time and depth on May 8 .

The calm hour and the shaken period, between 08:00 and 15:00, on May 8 is the focus of our modeling analysis. During this period, internal tides and solitons appear to have opposing effects on the acoustic propagation. While the internal tides during this period was observed to compress the sound-speed profile at the source location, thus tended to focus the energy into the lower layer, the solitons appeared to scatter the energy upward. Modeling results to be discussed in the next section suggest that, in addition to the diffusion of low-mode acoustic energy into the intermediate modes that filled the intermediate layer and above, scattering of the high-mode acoustic energy into the intermediate modes also occurred. 


\section{$\underline{\text { Before }}$ entrance of huge soliton}

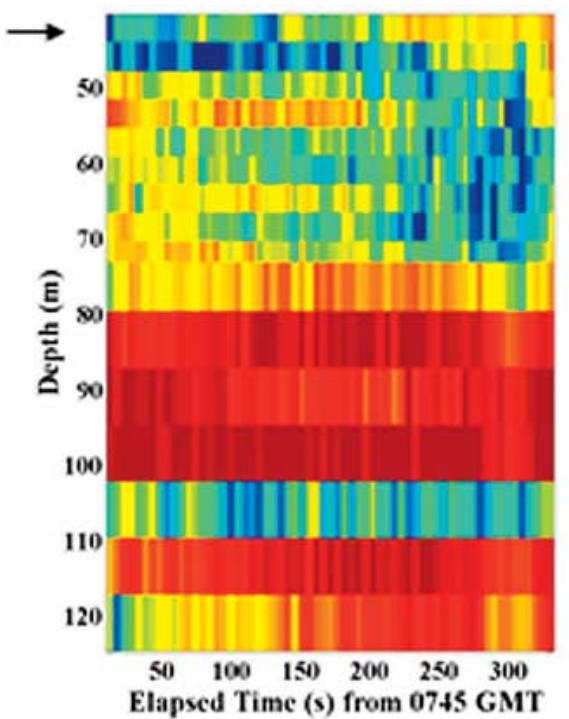

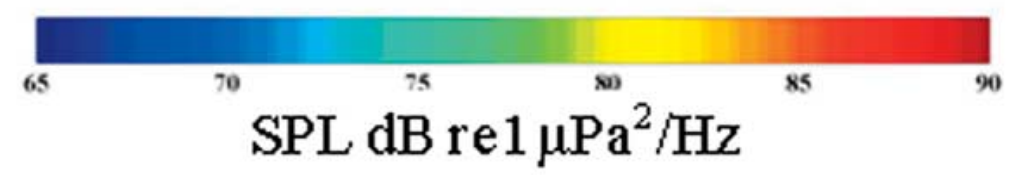

Fig. 11. Narrow-band (1-Hz bin at $400 \mathrm{~Hz}$ ) sound intensity level across the VLA, measured right before (left) and right after (right) the entrance of the leading, huge soliton into the cross-shelf transmission path on May 8 .

\section{Modeling Results}

The acoustic propagation model used in this study is that of Chiu et al. [10], [11]. Briefly, it is based on the physics of coupled normal modes. The basic formulation of the model involves decomposing the acoustic pressure into complex envelopes that modulate (mode by mode) analytic, rapidly varying, adiabaticmode solutions. Given sound speed, density, attenuation rate, and bathymetry as a function of space at every time increment, the acoustic solution is thus obtained by integrating a coupled set of differential equations governing these complex modal envelopes. Model output contains sound pressure, transmission loss, and phase and amplitude of the individual acoustic modes.

The acoustic modeling requires a realistic representation of the sound-speed field that is continuous in both space and time. To arrive at this, we introduced a dispersive empirical model for the perturbation field $\delta c^{(m)}$. Accounting for only the perturbations in the lowest EOF $f_{1}(r, z)$ and initialized by the corresponding mode-amplitude time series $a_{1}^{(o)}\left(t-t_{o}\right)$ observed by $\mathrm{S} 7$ at the range $r_{0}$, this model is given as

$$
\begin{aligned}
\delta c^{(m)}(r, z, t) & =\alpha \cdot a_{1}^{(0)}\left(\beta\left(t-t_{0}\right)-\frac{1}{c_{W}} \int_{\gamma_{0}}^{\gamma} \beta d r^{\prime}\right) \cdot f_{1}(r, z) \\
\alpha & =\left(\frac{h(r)}{h_{0}}\right)^{\nu}, \quad \beta=1+\mu \frac{h(r)-h_{0}}{h_{0}}
\end{aligned}
$$

where $c_{w}$ is the apparent internal wave speed along the transmission path and $\alpha$ and $\beta$ are the wave decay and stretching dispersion parameters, respectively, which are prescribed to depend on the ratio of the range-varying bathymetry $h(r)$ to the initial water depth $h_{0}$ at S7. With known bathymetry and $f_{1}(r, z)$ linearly interpolated from those at S7, S5, and S4 in vertical coordinates that are scaled by the local water depths, the model's free parameters are simply $\nu$ and $\mu$ and the $c_{w}$ s between S7 and S5 and between S5 and S4. (Note that the model gives nondispersive waves in the limit when both $\nu$ and $\mu$ approach zero.) The description is thus complete once these free parameters are tuned to obtain a good match between the model-evolved and observed perturbations at S5 and S4, respectively.

In the left column of Fig. 12, we show the modeled supertidal (peak) sound-speed perturbations between 08:00 and 15:00 on May 8 at five different isobaths along the transmission path. For comparison, the observed perturbations at S7, S5, and S4, respectively, are also displayed. Starting from S7 at the 348-m isobath, both data and model overlaps since the model is initialized there. Moving up the slope, the modeled waveform is seen to stretch and its amplitude lowered, as does the observed waveform. However, some misfits are found at S5 (226-m isobath) and S4 (120-m isobath). In particular, a new wave not part of the transbasin wave train is seen at S5. The model does not do as well on the shelf as the internal wavefield becomes very complex there [4]. Properly tuned, the same model (6) is also applicable to the perturbations in the tidal band. Similarly, in the right column of Fig. 12 we show the modeled and observed perturbations in this band between 08:00 and 15:00. Good agreement between data and model is found. Ignoring the small subtidal perturbations on May 8, the input sound-speed field to the acoustic model was taken to be the sum of the mean profile and the modeled perturbations in the tidal and supertidal bands.

In addition to the water-column sound speed, the acoustic modeling also requires the specification of the sediment's geoacoustic properties. For this, the sediment was assumed to be a homogenous layer with a sound speed of $1700 \mathrm{~m} / \mathrm{s}$, density of $1800 \mathrm{~kg} / \mathrm{m}^{3}$, and attenuation rate of $0.3 \mathrm{~dB} / \mathrm{m} / \mathrm{kHz}$. These num- 

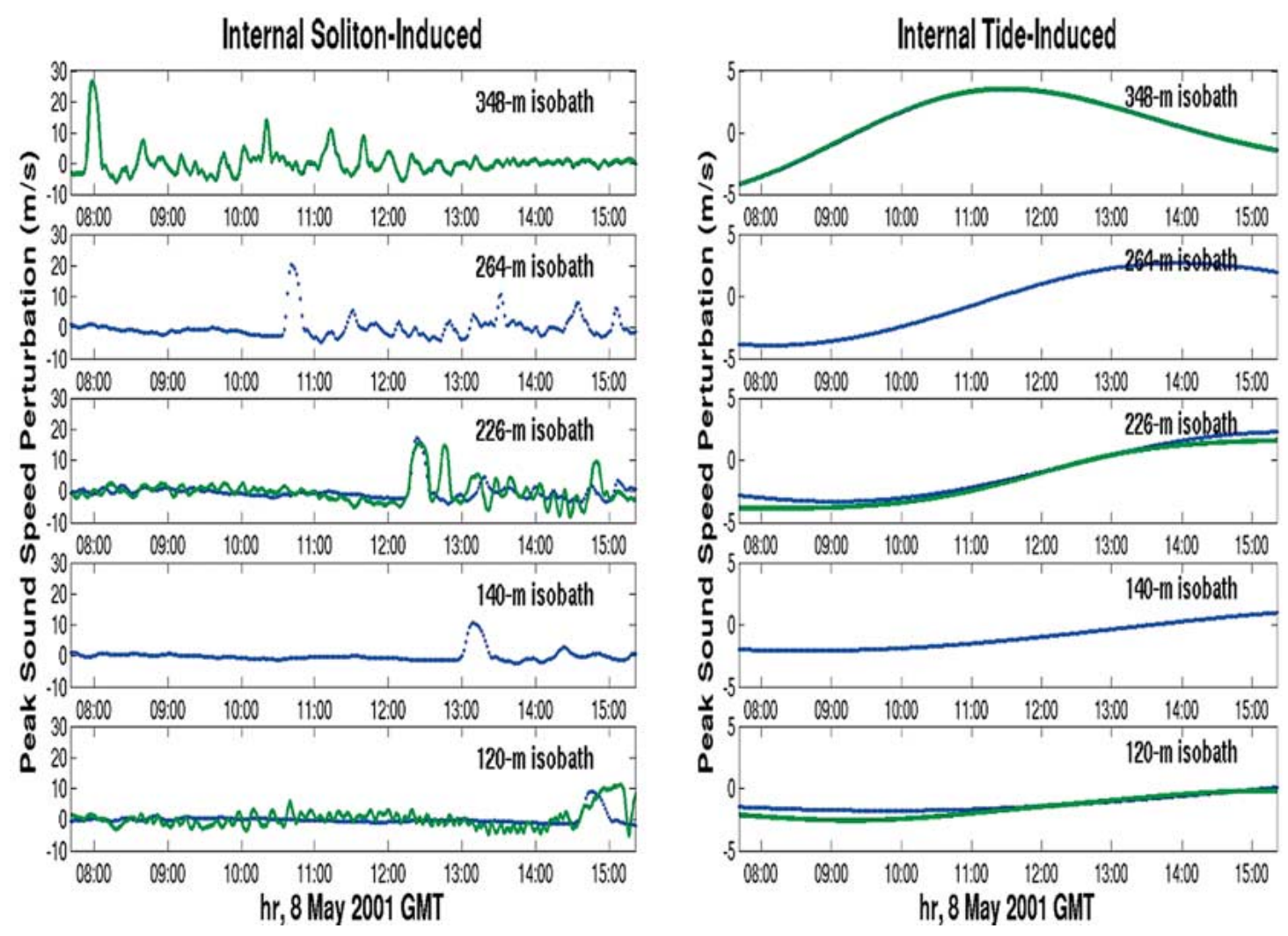

Fig. 12. Modeled (blue dots) and observed (green) time series of peak sound-speed change in the supertidal (left column) and tidal (right column) bands on May 8 at various isobaths along the upslope path.

bers correspond to nominal values deduced by Shock [12] from an initial analysis of the chirp-sonar data collected in a bottom survey along the transmission path. A more precise sediment model was not available at the time of this study. However, these simplified sediment parameters should be adequate for investigating intensity fluctuations caused by volume variability. The rapidly changing acoustic wavefield was computed at the carrier frequency $(400 \mathrm{~Hz})$ with a time step of approximately $40 \mathrm{~s}$; the results are presented next.

Fig. 13 shows three snapshots each of the modeled sound speed (left column), transmission loss TL (middle column), and relative acoustic modal magnitudes (right column). The top row contains the modeled fields right before the internal solitons enter the acoustic path. At this instant, the modeled sound speed is only mildly variable in range and the modeled TL contains three rather-structured interference patterns. The first is confined in the lower half of the water column and extends all the way from the source to the VLA. This bottom-trapped pattern is produced by the interference of a group of "low modes" (1-9). The second is associated with a group of "high modes" (20-38) that constructively interfere to produce approximately equally spaced shallow convergence zones at about $10 \mathrm{~m}$ below the sea surface on the slope. The intensity of these high modes disintegrates near the shelfbreak and on the shelf as a result of increased bottom loss and mode-coupling activities that are caused by the variable bathymetry. The third pattern, associated with the "very high modes" (52-68), starts out from the source as a very steep-angle beam. The beam then interacts with the surface and bottom a few times before stripped away by the shoaling bathymetry. Note that the "intermediate modes" (10-19) are not excited initially and, at this calm instant, mode couplings are activated primarily by the range-dependent bathymetry. The two relatively abrupt changes in the bathymetry, one close to the source and one at the shelfbreak, tend to transfer energy from higher to lower modes. This downward transfer is attributed to the fact that the angles of the modes are increasing in range at rates that are higher than twice the slope.

Comparing to the later snapshots shown in Fig. 13, the following remarks can be made. Before the entrance of the solitons, the modeled acoustic field on the shelf is dominated by two of the lowest modes ( 1 and 3 ), which focus the acoustic energy to depths below $70 \mathrm{~m}$. They also combine to form a quasi-acoustic null near $100 \mathrm{~m}$. After the entrance of the solitons, the structure of the modeled acoustic field becomes more vertically diffused at ranges beyond the leading soliton. In particular, the modeled acoustic energy on the shelf is no longer primarily trapped in the lower layer, but is diffused into the shallower depths. These modeling results are entirely consistent with the measured intensity data presented in the last section. The modal decomposition of the modeled acoustic field further reveals that the apparent diffusion is caused not only by the scattering of low-mode energy, but also by the infusion of high-mode energy into the intermediate modes. These coupling activities take place inside the soliton packet and reach a climax within the leading huge soliton where sound speed is severely range dependent. Span- 

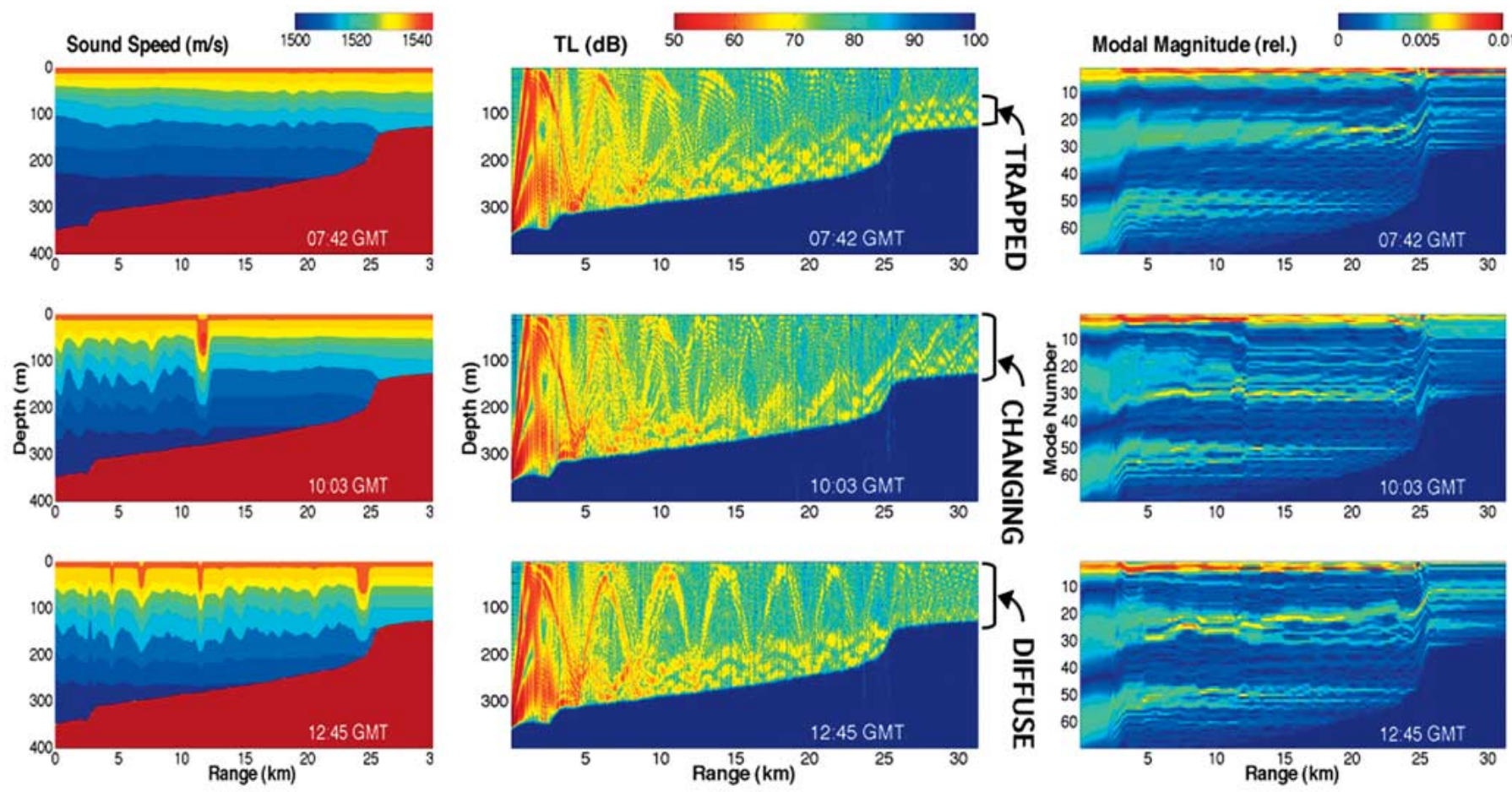

Transmission Loss

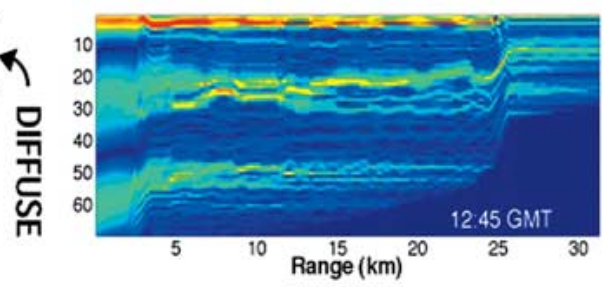

Sound Speed

\section{[Data-based empirical model]}

\section{Modal Magnitude}

Fig. 13. Three snapshots each of the modeled sound speed (left column), 400-Hz transmission loss (middle column), and relative acoustic modal magnitudes (right column).

ning the entire water column, the newly energized intermediate modes attenuate slowly in range on the shelf.

The modal decomposition of the modeled acoustic wavefields also shows that the transfer of the modal energy, caused by the solitons, is through cascading or ascending "thin paths" in the mode number-range space. This is indicative of a tight coupling, i.e., at each range, the resultant mode-mode interactions only take place between close neighbors. Insights into the tightness of the coupling can be obtained through an examination of the coupling coefficients in the coupled equations governing the modal amplitudes. After some algebraic manipulation similar to that presented by Milder [13] and Rutherford [14], the coupling coefficient between modes $n$ and $m$ as a function of range can be expressed as

$$
\begin{aligned}
\gamma_{m n}=\frac{-4 \omega^{2}}{k_{m}^{2}-k_{n}^{2}} \int_{0}^{\infty} \frac{1}{\rho \bar{c}^{3}} & \frac{\partial c}{\partial r} Z_{n} Z_{m} d z \\
& +\left.\left(\frac{1}{\rho_{w}}-\frac{1}{\rho_{s}}\right)\left(Z_{n} \frac{d h}{d r} Z_{m}\right)\right|_{z=h}
\end{aligned}
$$

where $\omega$ is the angular frequency of the transmission, $\rho$ is the density profile that changes from the water density $\rho_{\mathrm{w}}$ to the sediment density $\rho_{\mathrm{s}}$ at the interface $h$, and $k_{m}$ and $k_{n}$ are the horizontal wavenumbers of the $n$th and $m$ th normal modes $Z_{m}$ and $Z_{n}$, respectively. In this form, the effects of the water column and bathymetry on the mode-coupling coefficients are separated, with the first term representing the direct contribution

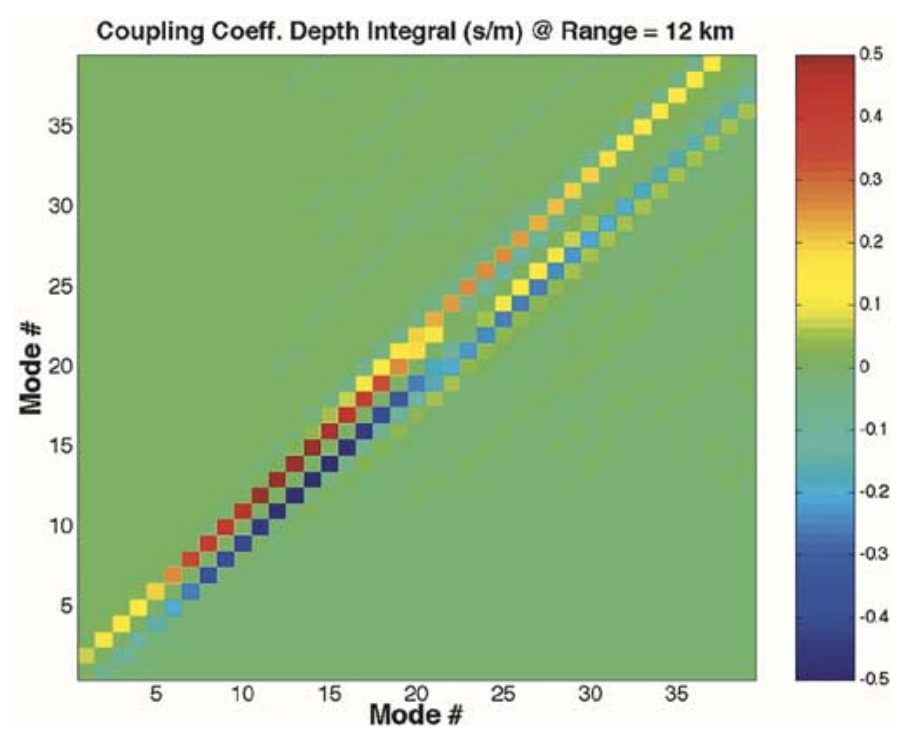

Fig. 14. Matrix of normalized mode-coupling coefficients at a range of $12 \mathrm{~km}$ resulting from an internal soliton.

from the range-varying water-column sound speeds. Assuming that the disturbances induced by an individual soliton located at $r_{0}$ has approximately a $\operatorname{sech}^{2}$ shape in range, a maximum of $\delta$, and a vertical structure $f_{1}$ unchanged over its full-width $\Delta$, i.e.,

$$
\delta c \approx \delta \operatorname{sech}^{2}\left(2 \pi \frac{r-r_{0}}{\Delta}\right) f_{1}\left(r_{o}, z\right)
$$



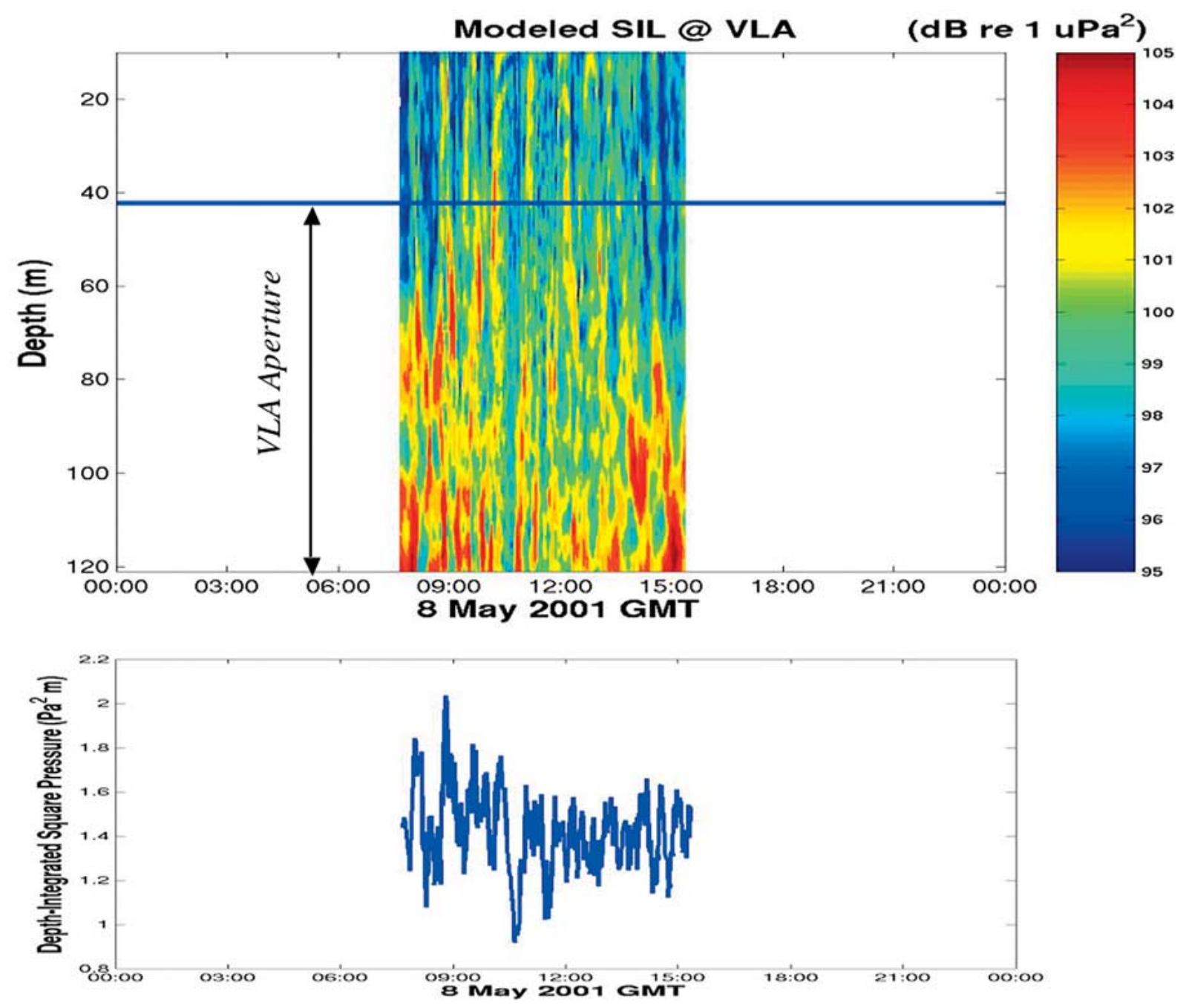

Fig. 15. Model sound-intensity pattern at the VLA location on May 8 between 07:15-15:00. Note that the leading soliton enters the acoustic path at 08:00 and reaches the VLA at approximately 15:00.

the first term in (7) can be recast, locally near $r_{0}$, as

$$
\begin{aligned}
\gamma_{m n}(\text { soliton })= & 2 \pi \frac{\delta}{\Delta} \operatorname{sech}\left(2 \pi \frac{r-r_{o}}{\Delta}\right) \\
& \cdot \tanh \left(2 \pi \frac{r-r_{o}}{\Delta}\right) C_{m n}(r) \\
C_{m n}= & \frac{-8 \omega^{2}}{k_{m}^{2}-k_{n}^{2}} \int_{0}^{\infty} \frac{f_{1}}{\rho \bar{c}^{3}} Z_{n} Z_{m} d z
\end{aligned}
$$

where the depth integrals $C_{m n}$ represent a matrix indicator that quantifies the degree of interactions between the different modes. In Fig. 14, we display the computed $C_{m n}$ at a range of $12 \mathrm{~km}$, which show the tightness of the coupling that is also common to all other ranges along the path. It is easily seen from Fig. 14 that mode coupling is restricted to the immediate neighbors, first neighbors for the low and intermediate modes and first and second neighbors for the high modes at a given range. In (9), the mathematical expression for $C_{m n}$ reveals that this restriction is imposed by the vertical structure $f_{1}$ of the solitons.
While Fig. 13 shows the modeled spatial variability of the acoustic field at three different times, Fig. 15 shows the modeled temporal acoustic variability (continuous in time) at the VLA. To quickly synthesize the effects of the signal bandwidth, the SIL displayed in Fig. 15 was computed from local range averages of the squared pressures modeled at the carrier frequency [15]. Similarly, these model results show that right before 08:00 (a period of calm), the acoustic energy is trapped in the lower layer (below $70 \mathrm{~m}$ ), but right after the entrance of solitons, the infusions of low- and high-mode energy into the intermediate modes that span the entire water column begin. It is interesting to note that the modeled intensity pattern shows a vertical distribution that is increasingly more diffused as the soliton packet moves upslope and that this apparent diffusion reaches a maximum as the packet arrive near the shelfbreak at about 13:00. This modeled phenomenon is linked to the strengthening of mode coupling, which can be shown, in theory, to depend on the ratio between the width of the soliton and the mode interference wavelength. The dependence is that mode-coupling activity increases as this ratio increases and reaches a maximum when the ratio approaches approximately unity. An examination 

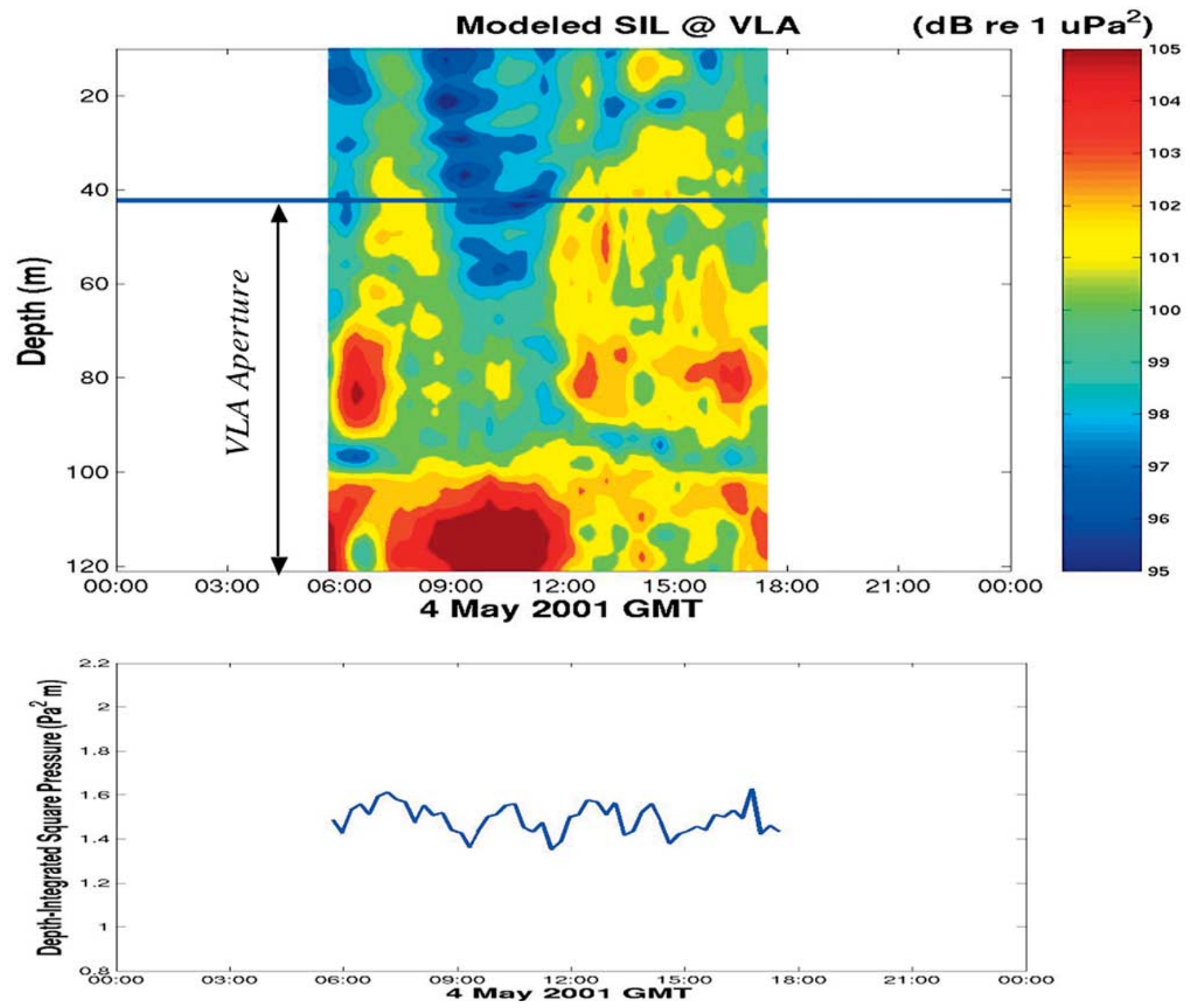

Fig. 16. Model sound intensity pattern at the VLA location on May 4 between 06:00-17:00.

of the interference wavelengths of the tightly coupled neighboring modes suggest that this is the case here, with the interference wavelengths decrease shoreward and reach about $1 \mathrm{~km}$, approximately the widths of the solitons, near the shelfbreak. This phenomenon of mode-coupling strengthening, however, is not observed in the data. The aperture of the VLA, spanning only two thirds of the water column, might have made such an observation difficult.

To obtain an appreciation of whether or not the acoustic energy is conserved in the water column during the passage of the solitons, the modeled sound pressure was squared and then integrated in depth over the entire water column at the VLA location. This depth-integrated squared pressure is displayed in the bottom panel of Fig. 15 as a function of time, showing significant rapid changes in the total energy in the water column. This, however, is not the case for May 4.

Modeling studies for May 4 have also been performed for a slightly extended time period using the same techniques de- scribed before, with a couple exceptions. First, neglecting internal waves, only internal tide-induced sound-speed perturbations were modeled. Second, expecting a slower time scale in intensity fluctuation, the acoustic model was run with a coarser time step of $30 \mathrm{~min}$. The extension of the modeling time window ( $2 \mathrm{~h}$ in front and $2 \mathrm{~h}$ behind) was necessary to clearly illustrate the cycling structure in the intensity pattern (top panel of Fig. 16). In contrast to May 8, the May 4 modeling results show that the internal tides modulate (stretch and compress) the sound channel, resulting in gradual shifts in the initial modal or angular distribution of the acoustic energy. These shifts in the excitation are manifested as slow vertical movements in the intensity pattern. Analogous to the measured data, the modeled pattern shows two high-intensity zones that slowly fade in and out in the lower layer and one in the upper layer that cycles up and down. The internal tides are found to add very little mode coupling to those produced by the variable bathymetry. The depth-integrated energy in the water column in the model, 
shown in the bottom panel of Fig. 16, also approximately conserves in May 4. It is important to note that the modeled period excludes the anomalously silent period aforementioned.

\section{CONCLUSION}

The temporal and spatial structures of the cross-shelf soundspeed field were analyzed from moored temperature data using temporal filtering to first delineate the perturbations in the subtidal, tidal, and supertidal bands, followed by principle-component decompositions to capture dominant vertical structures. The results show that the vertical structure in each of the three bands was dominated by two empirical functions throughout the entire experiment and that May 4 and 8 have significantly different environmental conditions, dominated by internal tides and solitons, respectively, leading to significantly different characteristics in the observed sound-intensity field.

As observed on May 8, the acoustic signal intensity on the shelf generally increased in the upper water column, but decreased in the lower water column as the internal solitons entered and moved along the acoustic path. This is indicative of a vertical redistribution of the acoustic energy. This apparent diffusion/infusion of sound energy can be explained by coupledmode physics: The low- and high-mode energies are scattered into the intermediate modes that span the entire water column and attenuate slowly on the shelf. At any given range, our modeling results also show that mode coupling is restricted to the immediate neighbors, resulting in gradual cascades or ascends of modal energy in range. The vertical structure of sound-speed change is responsible for this tight coupling behavior.

The internal tides on May 4 modulated (stretched and compressed) the sound channel, resulting in relatively slow vertical shifts in the intensity pattern. Unlike the huge internal solitons, the corresponding modeling results show that the internal tides produce little "additional" mode coupling with the depth-integrated energy approximately conserves.

\section{ACKNOWLEDGMENT}

The authors would like to thank every scientist, engineer, technician, student, and crew member who have participated in the ASIAEX SCS experiment. Their great efforts have collectively made this international experiment a major success. Special thanks go to J. Kemp and K. von der Heydt, who were instrumental to the design, development, and deployment of the L-shaped array that collected $600 \mathrm{~GB}$ of high-quality acoustic data. Finally, this work would not have been possible without the creativity of Dr. J. Simmen.

\section{REFERENCES}

[1] S. R. Ramp, J. F. Lynch, P. H. Dahl, C.-S. Chiu, and J. A. Simmen, "ASIAEX fosters advances in shallow-water acosutics," EOS, Trans. $A G U$, vol. 84, no. 37, pp. 361-367, 2003.
[2] J. Apel, M. Badiey, C.-S. Chiu, S. Finnette, R. Headrick, J. Kemp, J. Lynch, A. Newhall, M. Orr, B. Pasewark, D. Tielbuerger, A. Turgut, K. von der Heydt, and S. Wolf, "An overview of the 1995 SWARM shallow water internal wave acoustic scattering experiment," IEEE J. Oceanic Eng., vol. 22, pp. 465-500, July 1997.

[3] J. F. Lynch, A. E. Newhall, B. Sperry, G. Gawarkiewicz, A. Fredricks, P. Tyack, C.-S. Chiu, and P. Abbot, "Spatial and temporal variations in acoustic propagation characteristics at the New England shelfbreak front," IEEE J. Oceanic Eng., vol. 28, pp. 129-150, Jan. 2003.

[4] S. R. Ramp, T.-Y. Tang, T. F. Duda, J. F. Lynch, A. K. Liu, C.-S. Chiu, F. Bahr, H.-R. Kim, and Y.-J. Yang, "Internal solitons in the Northeastern South China Sea Part I: Sources and deep water propagation," IEEE J. Oceanic Eng., vol. 29, pp. 1157-1181, Oct. 2004.

[5] M. H. Orr, B. H. Pasewark, S. N. Wolf, J. F. Lynch, T. Schroeder, and C.-S. Chiu, "Impact of the South China Sea shelf break oceanographic variability on acoustic signal properties and conventional beamformer response," IEEE J. Oceanic Eng., vol. 29, pp. 1292-1307, Oct. 2004.

[6] T. F. Duda, J. F. Lynch, L. Wu, and C.-S. Chiu, "Fluctuations of 400 Hz sound intensity in the 2001 ASIAEX South China Sea experiment," IEEE J. Oceanic Eng., vol. 29, pp. 1264-1279, Oct. 2004.

[7] R.-C. Wei, "Bathymetric survey in South China Sea for ASIAEX," Inst. Undersea Technol., National Sun Yat-Sen Univ., Cruise Rep., June 2000.

[8] H. Medwin, "Speed of sound in water: A simple equation for realistic parameters," J. Acoust. Soc. Amer., vol. 58, pp. 1318-1319, 1975.

[9] G. Gawarkiewicz, J. Wang, M. Caruso, S. Ramp, K. Brink, and F. Bahr, "Shelfbreak circulation and thermohaline structure in the Northern South China Sea-Contrasting spring 2000 and spring 2001," IEEE J. Oceanic Eng., vol. 29, pp. 1131-1143, Oct. 2004.

[10] C.-S. Chiu, J. H. Miller, W. W. Denner, and J. F. Lynch, "A three-dimensional, broadband, coupled normal-mode sound propagation modeling approach," in Full Field Inversion Methods in Ocean and Seismic Acoustics, O. Diachok, A. Caiti, P. Gerstoft, and H. Schmidt, Eds. Boston, MA: Kluwer, 1995, pp. 57-62.

[11] C.-S. Chiu, J. H. Miller, and J. F. Lynch, "Forward coupled-mode propagation modeling for coastal acoustic tomography," J. Acoust. Soc. Amer, vol. 99, no. 2, pp. 793-802, 1996.

[12] S. C. Schock, "Remote prediction of the physical and acoustic properties of sediments in the South China Sea using a chirp sonar and biot theory," IEEE J. Oceanic Eng., vol. 29, pp. 1218-1230, Oct. 2004.

[13] D. M. Milder, "Ray and wave invariants for SOFAR channel propagation," J. Acoust. Soc. Amer., vol. 46, pp. 1259-1263, 1969.

[14] S. R. Rutherford, "An examination of coupled mode theory as applied to underwater sound propagation," Ph.D. dissertation, The Univ. Texas, Austin, 1979.

[15] C. H. Harrison and J. A. Harrison, "A simple relationship between frequency and range averages for broadband sonar," J. Acoust. Soc. Amer., vol. 97, no. 2, pp. 1314-1317, 1995.

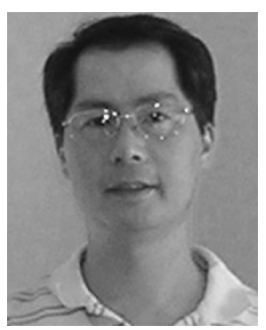

Ching-Sang Chiu received the B.S. degree in electrical engineering from Northeastern University, Boston, MA, in 1979 and the Sc.D. degree in oceanographic engineering from the Massachusetts Institute of Technology, Cambridge/Woods Hole Oceanographic Institution, Woods Hole, MA, joint program in 1985.

$\mathrm{He}$ is a Professor of oceanography at the Naval Postgraduate School, Monterey, CA. His research interests include ocean acoustics, acoustical oceanography, and coastal ocean processes and their influence on acoustic prediction. He has authored or coauthored more than 40 refereed publications in those subject areas.

Dr. Chiu is a Fellow of the Acoustical Society of America and Editor-in-Chief of the Journal of Computational Acoustics. 


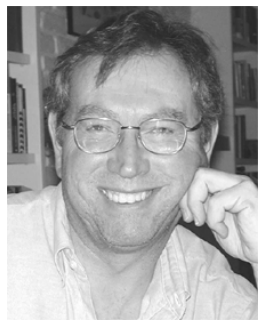

Steven R. Ramp received the M.S. degree in physical oceanography from the University of Washington, Seattle, in 1976, and the Ph.D. degree in physical oceanography from the Graduate School of Oceanography, University of Rhode Island, Narragansett, RI, in 1986

Since 1986, he has been with the U.S. Navy as a Professor at the Naval Postgraduate School, Monterey, CA, and a Program Officer at the Office of Naval Research, Arlington, VA. Prior to this, he spent time at the National Marine Fisheries Service, Woods Hole, MA. His research specialty is ocean observations from both ships and oceanographic moorings, and has organized major expeditions to the Japan Sea, East China Sea, and South China Sea. He was the International Scientific Coordinator for the Asian Seas International Acoustics Experiment (ASIAEX).

Christopher W. Miller (S'96-M'96) received the B.S. degree in electronics engineering from California Polytechnic State University, San Luis Obispo, in 1991 and the M.S. degree in electrical engineering from the Naval Postgraduate School, Monterey, CA, in 1998.

Since 1992, he has been with the Naval Postgraduate School and currently is a Research Associate with the Oceanography Department, where he is involved with data collection, analysis, and programming development for acoustical oceanography experiments.

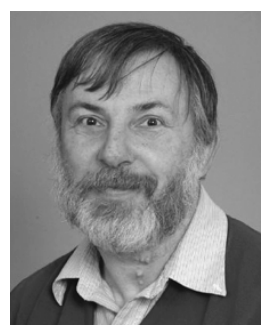

James F. Lynch (M'96-SM'03) was born in Jersey City, NJ, on June 3, 1950. He received the B.S. degree in physics from Stevens Institute of Technology, Hoboken, NJ, in 1972 and the Ph.D. degree in physics from the University of Texas, Austin, in 1978.

He was with the Applied Research Laboratories, University of Texas at Austin (ARL/UT) from 1978 to 1981 , after which he joined the scientific staff at the Woods Hole Oceanographic Institution (WHOI), Woods Hole, MA. He has been with WHOI since then and currently holds the position of Senior Scientist in the Applied Ocean Physics and Engineering Department. His research specialty areas are ocean acoustics and acoustical oceanography, but he also greatly enjoys occasional forays into physical oceanography, marine geology, and marine biology.

Dr. Lynch is a Fellow of the Acoustical Society of America and Editor-inChief of the IEEE JOURNAL OF OCEANIC ENGINEERING.

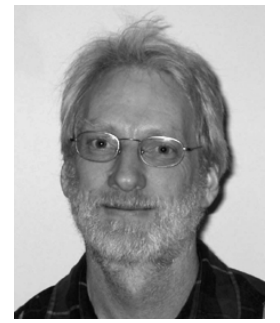

Timothy F. Duda received the B.A. degree in physics from Pomona College in 1979 and the Ph.D. degree in oceanography from the Scripps Institution of Oceanography, University of California, San Diego, in 1986.

$\mathrm{He}$ has been a Scientist with the Woods Hole Oceanographic Institution, Woods Hole, MA, since 1991. Prior to that, he held positions at the University of California, Santa Cruz. His three primary fields of study are ocean acoustic propagation, ocean internal gravity waves, and ocean mixing processes. $\mathrm{He}$ has modeled and analyzed fluctuations of ocean acoustic transmissions; has made electromagnetic-type internal-wave measurements; and performed in situ measurements of ocean microstructure.

Dr. Duda is a member of the American Meteorological Society, the American Geophysical Union, and the Acoustical Society of America.

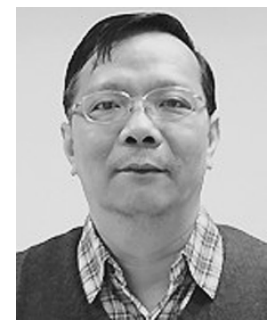

Tswen Yung Tang was born in Taiwan in 1952. He received the M.S. degree in physical oceanography from National Taiwan University, Taiwan, in 1975 and the Ph.D. degree in physical oceanography from North Carolina State University, Raleigh, in 1984.

In August 1993, he was appointed Professor of Physical Oceanography at the Institute of Oceanography, National Taiwan University. He was appointed Program Manager of the Division of Marine Science, National Science Council, Taiwan, in January 2004. His research interests include equatorial dynamics, variability of upstream of Kuroshio, Kuroshio intrusion at Luzon Strait, circulation in the South China Sea, and internal waves. 